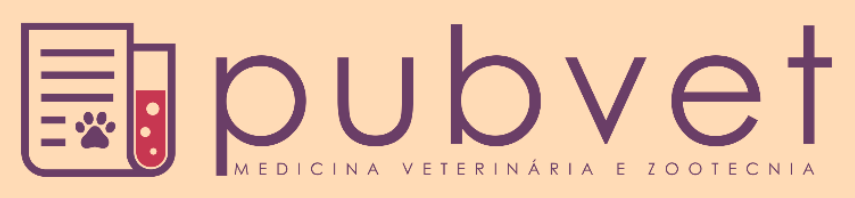

https://doi.org/10.31533/pubvet.v12n10a181.1-5

\title{
Eficácia de oxitetraciclina no tratamento de Streptococus Agalactiae em tilápia a diferentes temperaturas de criação
}

\author{
Iris de Fatima Mariotto ${ }^{1}$, Sheila Rezler Wosiacki² ${ }^{\ominus}$, Marcos Ferrante M $^{2 *} \bullet$ \\ ${ }^{I}$ Graduanda do curso de Medicina Veterinária da Universidade Estadual de Maringá, Umuarama, PR Brasil. \\ ${ }^{2}$ Docente do curso de Medicina Veterinária da Universidade Estadual de Maringá, Umuarama, PR Brasil. \\ *Autor para correspondência: marcosferrante@gmail.com
}

RESUMO. A estreptococoses é uma das principais causadoras de morbidade e mortalidade na tilapicultura do Brasil e a oxitetraciclina é um dos principais antibióticos utilizado por décadas no seu tratamento. Existem estudos que determinam as diferenças na farmacocinética de oxitetraciclina em Tilápia do Nilo segundo as temperaturas de criação. Porém, não existem estudos que avaliem o impacto dessas mudanças na temperatura de criação sobre a eficácia clínica da oxitetraciclina nas doses habituais. Este estudo foi realizado para avaliar o efeito de mudanças na temperatura de criação nas taxas de mortalidade de animais portadores e de cura nas infecções por Streptococcus Agalactiae. $O$ índice farmacocinético/farmacodinâmico (PK/PD) utilizado para a determinação de eficácia foi a área sob a curva da concentração plasmática de oxitetraciclina em função do tempo (ASC)/concentração inibitória mínima (CIM). Para estimação das taxas de animais curados, carreadores e mortos foi realizada uma simulação de Monte Carlo da ASC seguidamente do modelamento PK/PD. Foram observadas mudanças nas frequências de indivíduos mortos e de portadores estimados relacionadas com as variações na temperatura de criação. As taxas de mortalidade estimadas foram de $58 \%$ e $12 \%$ e as taxas de portadores assintomáticos foram de $11 \%$ e $19 \%$ segundo as temperaturas de criação de $25^{\circ} \mathrm{C}$ e $30^{\circ} \mathrm{C}$, respectivamente. A probabilidade de obter a cura bacteriológica após o tratamento aumentou para os tratamentos realizados com temperatura de criação de $30^{\circ} \mathrm{C}$. Pequenos incrementos na CIM determinaram grandes mudanças nas taxas de animais curados, carreadores e mortos. Isso deixa em evidencia a necessidade de incorporar no protocolo terapêutico o isolamento bacteriológico, a determinação da CIM e otimização de dose terapêutica segundo a temperatura de criação a fim de evitar falhas terapêuticas e consequentemente potencializar o desenvolvimento de resistência.

Palavras chave: estreptococose, farmacodinâmica, farmacocinética, tetraciclina, tilapicultura

\section{Efficacy of Oxytetracycline in the treatment of Streptococus agalactiae in tilapia at different breeding temperatures}

ABSTRACT. Streptococcosis is one of the main causes of morbidity and mortality in
Brazilian tilapia farming and oxytetracycline is one of the main antibiotics that have been
used for decades in its treatment. There are studies that determine the differences in the
pharmacokinetics of oxytetracycline in Nile Tilapia according to breeding temperatures.
However, there are no studies evaluating the impact of these changes in breeding
temperature on the clinical efficacy of oxytetracycline at usual doses. The objective of this
study was to evaluate the effect of changes in breeding temperature on mortality rates,
carrier animals and cure in Streptococcus agalactiae infections. The pharmacokinetic /
pharmacodynamic index (PK/PD) used for the determination of efficacy was the area below
the plasma concentration of oxytetracycline versus time (AUC)/minimum inhibitory 
concentration (MIC) curve. A Monte Carlo simulation of the AUC followed by the PK/PD modeling was used to estimate the rates of cured, carrion and dead animals. Changes were observed in the frequencies of dead individuals and estimated carriers related to variations in breeding temperature. The estimated mortality rates were $58 \%$ and $12 \%$ and the rates of asymptomatic carriers were $11 \%$ and $19 \%$, respectively, according to the breeding temperatures of $25^{\circ} \mathrm{C}$ and $30^{\circ} \mathrm{C}$. The probability of obtaining bacteriology cure after treatment increased for treatments performed at $30^{\circ} \mathrm{C}$ breeding temperature. It was observed that small increments in the CIM determined large changes in the rates of cured, carcass and dead animals. This demonstrates the need to incorporate in the therapeutic protocol bacteriological isolation, MIC determination and optimization of therapeutic doses in order to avoid therapeutic failures and consequently potentiate the development of resistance.

Keywords: pharmacodinamic, pharmacokinetic, streptococoses, tetracycline, tilapiculture

\section{Eficacia de Oxitetraciclina en el tratamiento de Streptococus Agalactiae en Tilapia del Nilo a diferentes temperaturas de cría}

RESUMEN. La estreptococosis es una de las principales causantes de morbilidad y mortalidad en la producción de Tilapia de Brasil y la oxitetraciclina es uno de los principales antibióticos que viene siendo utilizado por décadas en su tratamiento. Existen estudios que determinan las diferencias en la farmacocinética de oxitetraciclina en Tilapia del Nilo según las temperaturas de cría. Sin embargo, no existen estudios que evalúen el impacto de estos cambios en la temperatura de cría sobre la eficacia clínica de la oxitetraciclina en las dosis habituales. El objetivo de este estudio fue evaluar el efecto de cambios en la temperatura de cría en las tasas de mortalidad, de animales portadores y de curación en las infecciones por Streptococcus Agalactiae. El índice farmacocinético/farmacodinámico (PK/PD) utilizado para la determinación de eficacia fue el área bajo de la curva de la concentración plasmática de oxitetraciclina en función del tiempo (ABC)/concentración inhibitoria mínima (CIM). Para la estimación de las tasas de animales curados, transportadores y muertos se realizó una simulación de Monte Carlo del ABC seguida del modelado PK/PD. Se observaron cambios en las frecuencias de individuos muertos y de portadores estimados relacionados con las variaciones en la temperatura de cría. Las tasas de mortalidad estimadas fueron de $58 \%$ y $12 \%$ y las tasas de portadores asintomáticos fueron del $11 \%$ y $19 \%$ según las temperaturas de cría de $25^{\circ} \mathrm{C} \mathrm{y}$ $30^{\circ} \mathrm{C}$, respectivamente. La probabilidad de obtener la cura bacteriológica después del tratamiento aumento significativamente para los tratamientos realizados con temperatura de cría de $30^{\circ} \mathrm{C}$. Pequeños incrementos en la CIM determinan grandes cambios en las tasas de animales curados, transportadores y muertos. Esto deja en evidencia la necesidad de incorporar en el protocolo terapéutico el aislamiento bacteriológico, la determinación de la CIM y optimización de dosis terapéutica según la temperatura de cría a fin de evitar fallas terapéuticas y consecuentemente potenciar el desarrollo de resistencia.

Palabras claves: estreptococosis, farmacocinética, farmacodinámica, tetraciclinas, cria de tilapia

\section{Introdução}

A Tilápia do Nilo, Oerochromis niloticus, espécie pouco valorizada até meados da década de 90, hoje está entre as espécies de peixes mais cultivadas mundialmente (FAO, 2013), assumindo o primeiro lugar das espécies mais produzidas pela aquicultura continental no Brasil (MAPA 2012). Por outro lado, a infecção por Streptococcus agalactiae é uma grande ameaça para a Tilápia do Nilo, na aquicultura em todo o mundo. Este tipo de estreptococose provoca septicemia aguda e meningoencefalite. A terapia com antibióticos é o método indicado para controlar surtos, de preferência com a utilização de vacinas (Faria et al., 2014).

A oxitetraciclina, antibiótico do grupo das tetraciclinas com largo espectro de ação, é o antibiótico preferido para o tratamento de doenças bacterianas na aquicultura, devido à sua eficácia relativa, baixo custo e por possuir a capacidade de 
difusão em fluidos e tecidos corpóreos (Rigos et al., 2003).

Os modelos PK/PD relacionam parâmetros farmacocinéticos (PK) com parâmetros farmacodinâmicos (PD) e são amplamente utilizados como indicadores de eficácia de tratamentos antibióticos em humanos e animais (EMA, 2016; Abdelraouf, et al. 2017), A utilização deste modelos conjuntamente com o método de simulação de Monte Carlo foi descrita por Dudley et al., (2000) e por Trang et al., (2017), e atualmente é a principal metodologia empregada para a avaliação e otimização de doses terapêuticas de antibióticos (EMA, 2016; Toutain 2016; Toutain et al., 2017)

Existem estudos que determinam as diferenças na farmacocinética de oxitetraciclina em Tilápia do Nilo segundo as temperaturas de criação (Chen et al. 2005). Porém não existem estudos que avaliem o impacto dessas mudanças na temperatura de criação sobre a eficácia clínica da oxitetraciclina nas doses habituais.

O objetivo deste estudo foi avaliar o efeito de mudanças na temperatura de criação nas taxas de mortalidade, de animais portadores e de cura nas infecções por Streptococcus Agalactiae utilizando em Tilápia do Nilo.

\section{Material e Métodos}

Realizou-se um modelamento PK/PD para a determinação da eficácia do tratamento com 100 $\mathrm{mg} / \mathrm{kg}$ de oxitetraciclina via oral a cada 12 horas de Tilapias do Nilo infectadas por cepas de Streptococcus Agalactia. O parâmetro de eficácia utilizado foi o índice da área sob da curva da concentração plasmática em função do tempo/concentração inibitória mínima(ASC/MIC) (Martinez et al., 2007; Ahamad et al., 2016).

Foi realizada uma simulação de Monte Carlo dos parâmetros farmacocinéticos de 10.000 indivíduos, seguidamente foi realizado modelamento PK/PD para determinar as taxas de animais curados, portadores e mortos baseados na metodologia descrita por Dudley et al. (2000) e Trang et al., (2017). A simulação dos parâmetros farmacocinéticos utilizados foi baseada nos dados do estudo realizado por Chen et al. (2005) (Gráfico 1). A seleção dos parâmetros farmacodinâmicos foi realizada a partir dos dados do estudo epidemiológico realizado por Gozi (2016) com bactérias isoladas de Tilápia do Nilo nos Estados de Minas Gerais, São Paulo e Paraná no Brasil (Grafico 2).
A análise estatística foi realizada no programa Excel e no programa BioEstat 5.0. Para a comparação das taxas de eficácia foi utilizado o teste de independência do Qui-Quadrado com uma significância de $(\mathrm{P}=0,05)$.

\section{Resultados e Discussão}

A oxitetraciclina é o principal antibiótico para o tratamento de infecções clínicas por estudos experimentais com cepas bacterianas clínicas demonstram alterações na sua eficácia (Faria et al., 2014; Chideroli et al., 2017). Complementarmente, o presente estudo demostra mudanças na temperatura de criação determinam alto impacto na eficácia terapêutica.

A temperatura de criação da Tilápia de Nilo determina diferença na concentração de oxcitetracilina no cérebro (Gráfico 1). Segundo Faria et al. (2014), a ineficácia do tratamento da estreptococoses em tilápia é evidenciada pelo isolamento do microrganismo no tecido cerebral, indicando a importância das concentrações do fármaco no sistema nervoso central para obter o sucesso terapêutico.

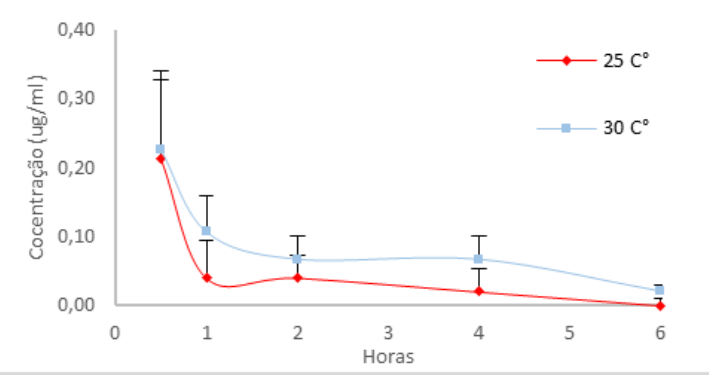

Gráfico 1. Farmacocinética de oxitetraciclina após a administração de $100 \mathrm{mg} / \mathrm{kg}$ via oral em Tilapias do Nilo submetidos às diferentes temperaturas de criação (Adaptado de Chen et al. 2005).

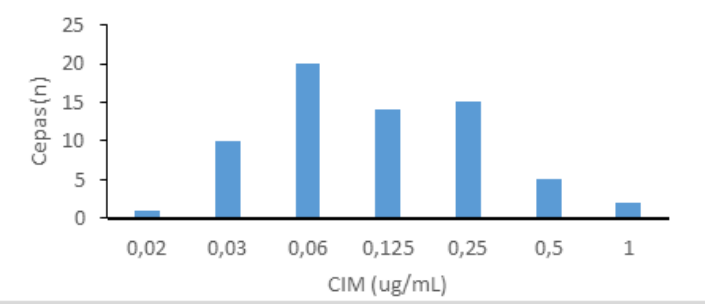

Gráfico 2. Distribuição da concentração mínima inibitória da oxitetraciclina para o grupo de StreptococcusAgalactiae (Adaptado de Gozi, 2016).

Segundo o estudo epidemiológico realizado por Gozi et al. (2016), o intervalo das CIM para oxitetraciclina foi de 0,2 a $1 \mu \mathrm{g} / \mathrm{mL}$ nos 67 
isolados, com uma maior prevalência na concentração $0,06 \mu \mathrm{g} / \mathrm{m}$ (Gráfico 2). Por tanto, neste estudo foi avaliado a relação entre as concentrações de oxitetraciclina de cérebro e as CIM de isolados no brasil.

A modelagem PK/PD permitiu estimar a eficácia do tratamento segundo as mudanças na temperatura de criação. Foram observadas mudanças nas frequências de indivíduos mortos e de portadores estimados relacionadas com as variações na temperatura de criação (Gráfico 3). As taxas de mortalidade estimadas foram de $58 \%$ e $12 \%$ e as taxas de portadores assintomáticos foram de $11 \%$ e $19 \%$ segundo as temperatura de criação de $25^{\circ} \mathrm{C}$ e $30^{\circ} \mathrm{C}$, respetivamente (Gráfico 4). A probabilidade de obter a cura bacteriologia após o tratamento aumentou para os tratamentos realizados com temperatura de criação de $30^{\circ} \mathrm{C}(\mathrm{P}$ $<0,001$ ) (Gráfico 4). Esses resultados indicam a necessidade de realizar ajustes nas doses terapêuticas que permitam obter taxas de eficácia adequadas.

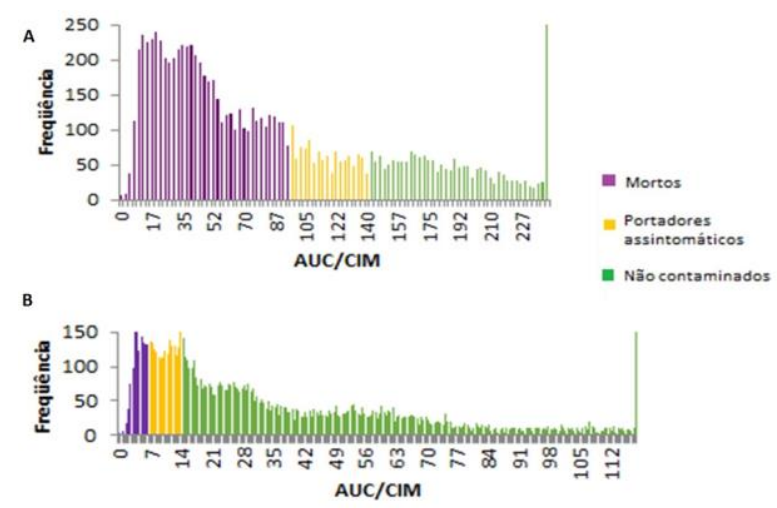

Gráfico 3. Frequência de indivíduos segundo a relação AUC/CIM de oxitetraciclina baseado na simulação de Monte Carlo $(\mathrm{n}=10.000)$. A) Temperatura de criação de $\left.25^{\circ} \mathrm{C} . \mathrm{B}\right)$ Temperatura de criação de $30^{\circ} \mathrm{C}$.

O sucesso terapêutico da antibioticoterapia depende principalmente da relação entre a concentração do fármaco no sítio de infeção com a concentração inibitória mínima da bactéria causante da infeção (Ahmad et al., 2016; Toutain et al., 2002). Por tanto, a falha terapêutica poderia ser de origem farmacocinético (PK), farmacodinâmica (PD) ou por uma combinação de ambos. As falhas terapêuticas de origem PK se apresentam quando o fármaco não atinge a concentração necessária no sítio de ação e as de origem PD quando a bactéria resiste as concentrações terapêuticas originais ( $\mathrm{Hu} \&$ Cheng 2016; Torneke \& Torren-do, 2015; Toutain et al., 2016).
A presente análise $\mathrm{PK} / \mathrm{PD}$ permite inferir que a oxitetraciclina poderia ser utilizada devido a que as bactérias apresentaram susceptibilidade; porém, para obter resultados de eficácia adequados deveram ser realizados os ajustes de doses correspondentes que permitam corrigir a falha farmacocinética dependente da temperatura de criação.

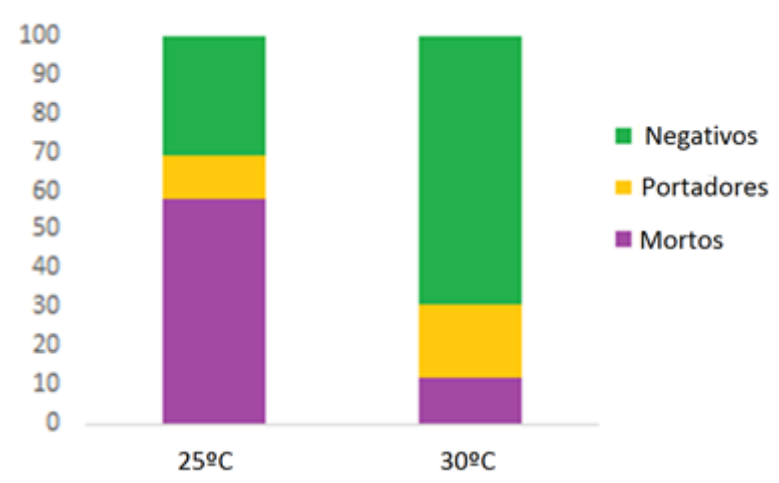

Gráfico 4: Probabilidade de obter animais curados, portadores e mortos após o tratamento de Streptococcus Agalactiae com oxitetraciclina $100 \mathrm{mg} / \mathrm{kg}$ via oral durante 10 dias em Tilápias do Nilo segundo a temperatura de criação, baseado na simulação de Monte Carlo $(\mathrm{n}=10.000)$.

Devido a que mudanças na temperatura da agua determinam alterações na farmacocinética, existem estudos que determinam ajustes no período de carência de antibióticos baseados na temperatura de criação, como no caso de florfenicol (Feng et al., 2008). Porém, o presente estudo demostrou que também são necessários mais estudos que permitam ajustar as doses terapêuticas segundo as temperaturas de criação.

\section{Conclusão}

Pequenos incrementos na concentração inibitória mínima determinam grandes mudanças nas taxas de animais curados, carreadores e mortos. Isso deixa em evidencia a necessidade de incorporar no protocolo terapêutico o isolamento bacteriológico, a determinação da concentração inibitória mínima e otimização de doses terapêutica segundo as temperaturas de criação a fim de evitar falhas terapêuticas e consequentemente potencializar o desenvolvimento de resistência.

\section{Referências}

Abdelraouf, K., Linder, K. E., Nicolau, D. P. \& Nailor, M. D. 2016. Predicting and Preventing Antimicrobial Resistance Utilizing Pharmacodynamics: Part I Gram Positive 
Bacteria. Expert opinion on drug metabolism \& toxicology 12(3), 267-80.

Ahmad, I., Huang, L., Hao, H., Sanders, P. \& Yuan, Z. 2016. Application of PK/PD Modeling in Veterinary Field: Dose Optimization and Drug Resistance Prediction. Bio Med Research International, ID 5465678.

BRASIL, Ministério da Agricultura, Pecuária e Abastecimento - MAPA. 2012. Boletim estatístico da pesca e aquicultura, Brasília, DF.

Chen, C.-Y., Wooster, G. A., Getchell, R. G. \& Bowser, P. R. 2005. Distribution and depletion of oxytetracycline in two warm-water fish: tilapia and hybrid striped bass. Journal of the World Aquaculture Society 36 (4), 564-569.

Chideroli, R. T. 2017. "Emergence of a New Multidrug-Resistant and Highly Virulent Serotype of Streptococcus Agalactiae in Fish Farms from Brazil." Aquaculture 479, 45-51.

Dudley, M. N. \& Ambrose, P. G. 2000. Pharmacodynamics in the study of drug resistance and establishing in vitro susceptibility breakpoints: ready for prime time. Current Opinion in Microbiology 3, 515521

European Medicine Agency - EMA. 2016. "Guideline on the Use of Pharmacokinetics and Pharmacodynamics in the Development of Antimicrobial Medicinal Products."

Faria, F. C., Leal, C. A. G., Carvalho-Castro, G. A., Leite, R. C., Figueredo, H. C. P. 2014. Carrier state induced by oxytetracycline therapy against streptococcosis in Nile tilapia, Oreochromis niloticus (L.). Journal of Fish Diseases, 37 (9), 853-857.

Feng, J.-B., Jia, X.-P., Li, L.-D., 2008. Tissue distribution and elimination of florfenicol in tilapia (Oreochromis niloticus $\times$ O. caureus) after a single oral administration in freshwater and seawater at $28^{\circ} \mathrm{C}$. Aquaculture 276, 29 35.

Food and Agriculture Organization of the Unite Nations - FAO. 2013. Fishery and aquaculture statistics, Rome, Italy. Geneva: 2015.

Gozi, K. S. 2016. Perfil de resistência à antimicrobianos de Aeromonas sp. e
Streptococcus $\mathrm{sp}$. isolados de tilápia-do-Nilo e detecção dos genes envolvidos na resistência à tetraciclina. - Universidade Estadual Paulista, Jacoticabal, 1-52

Hu, Y. \& Cheng, H. 2016. "Health Risk from Veterinary Antimicrobial Use in China's Food Animal Production and Its Reduction." Environmental Pollution 219, 993-97.

Martinez, M., Toutain, P. L., Bousquet-Me, A. 2007. "AUC/MIC: A PK/PD Index for Antibiotics with a Time Dimension or Simply a Dimensionless Scoring Factor?" J Antimicrob Chemother, 60 (6),1185-8.

Rigos. G., Nengas, I., Tyrpenou, A. E, Alexis M. \& Troisi G. M. 2003. "Farmacocinética e biodisponibilidade de oxitetraciclina em goraz gilthead (Sparus aurata) após uma dose única". Aquaculture, 221, 75-83.

Torneke, K. \& Torren-Edo, J. 2015. "The Management of Risk Arising from the Use of Antimicrobial Agents in Veterinary Medicine in EU/EEA Countries-a Review." Journal of Veterinary, Pharmacology and Therapy: 51928.

Toutain, P.L. 2016. "Veterinary Medicine Needs New Green Antimicrobial Drugs." Frontiers in Microbiology 7, 1196.

Toutain, P.L. 2017. "Standard PK/PD Concepts Can Be Applied to Determine a Dosage Regimen for a Macrolide: The Case of Tulathromycin in the Calf." Journal of Veterinary Pharmacology and Therapeutics 40(1)16-27.

Trang, M., Dudley, M., Bhavnani, S. M. 2017. Use of Monte Carlo simulation and considerations for PK-PD targets to support antibacterial dose selection. Current Opinion in Pharmacology $36: 107-113$.

Recebido: 15 Julho. 2018.

Aprovado: 15 Agosto. 2018

Publicado: 25 Setembro 2018

Licenciamento: Este artigo é publicado na modalidade Acesso Aberto sob a licença Creative Commons Atribuição 4.0 (CC-BY 4.0), a qual permite uso irrestrito, distribuição, reprodução em qualquer meio, desde que o autor e a fonte sejam devidamente creditados. 